\title{
Localization of the auxin permease AUX1 suggests two functionally distinct hormone transport pathways operate in the Arabidopsis root apex
}

\begin{abstract}
Ranjan Swarup, ${ }^{1,5}$ Jirí Friml, ${ }^{2,3,5}$ Alan Marchant, ${ }^{1}$ Karin Ljung, ${ }^{4}$ Goran Sandberg, ${ }^{4}$ Klaus Palme, ${ }^{2}$ and Malcolm Bennett ${ }^{1,6}$

${ }^{1}$ School of Biosciences, University of Nottingham, Nottingham NG7 2RD, UK; ${ }^{2}$ Max-Delbrück-Laboratorium in der Max-Planck-Gesellschaft, Carl-von-Linné-Weg 10, D-50829 Köln, Germany; ${ }^{3}$ Department of Biochemistry, Faculty of Science, Masaryk University, Kotlárská 2, 61137 Brno, Czech Republic; ${ }^{4}$ Department of Forest Genetics and Plant Physiology, The Swedish University of Agricultural Sciences, S-901 83, Umea, Sweden
\end{abstract}

\begin{abstract}
Auxins represent an important class of plant hormone that regulate plant development. Plants use specialized carrier proteins to transport the auxin indole-3-acetic acid (IAA) to target tissues. To date, efflux carrier-mediated polar auxin transport has been assumed to represent the sole mode of long distance IAA movement. Localization of the auxin permease AUX1 in the Arabidopsis root apex has revealed a novel phloem-based IAA transport pathway. AUX1, asymmetrically localized to the plasma membrane of root protophloem cells, is proposed to promote the acropetal, post-phloem movement of auxin to the root apex. MS analysis shows that IAA accumulation in aux1 mutant root apices is impaired, consistent with an AUX1 phloem unloading function. AUX1 localization to columella and lateral root cap tissues of the Arabidopsis root apex reveals that the auxin permease regulates a second IAA transport pathway. Expression studies using an auxin-regulated reporter suggest that AUX1 is necessary for root gravitropism by facilitating basipetal auxin transport to distal elongation zone tissues.
\end{abstract}

Received June 23, 2001; revised version accepted August 27, 2001.

Auxins represent important regulators of plant development (Davies 1995) and are unique among plant signaling molecules in being subject to polar transport (Lomax et al. 1995). Studies using auxin transport inhibitors have shown that the polarity of auxin movement provides an important developmental signal during embryogenesis (Liu et al. 1993), lateral root initiation (Casimiro et al. 2001), leaf vascular patterning (Mattsson et al. 1999), and

[Key Words: Auxin transport; phloem unloading; root gravitropism; AUX1; auxin influx carrier; membrane localization]

${ }^{5}$ These authors contributed equally to this work.

${ }^{6}$ Corresponding author.

E-MAIL Malcolm.Bennett@nottingham.ac.uk; FAX 44-115-951-6334. Article and publication are at http://www.genesdev.org/cgi/doi/10.1101/ gad.210501. root gravitropism (Marchant et al. 1999; Rashotte et al. 2000; Parry et al. 2001). Plants use specialized influx and efflux carriers to mobilize the major form of auxin, indole-3-acetic acid (IAA) from cell to cell (for review, see Palme and Gälweiler 1999). Molecular genetic studies in Arabidopsis thaliana have identified putative auxin influx and efflux carrier components encoded by the $A U X 1$ (Bennett et al. 1996) and AtPIN/AGR/EIR gene sequences (Chen et al. 1998; Gälweiler et al. 1998; Luschnig et al. 1998; Müller et al. 1998; Utsuno et al. 1998), respectively. AUX1 belongs to the auxin amino acid permease (AAAP) family of proton-driven transporters (Bennett et al. 1996), whereas AtPIN sequences exhibit homology to bacterial transporters (Palme and Gälweiler 1999|. Mutations in the Arabidopsis gene AtPIN1 disrupt polar auxin transport (Okada et al. 1991), embryo patterning (Liu et al. 1993), and vascular development (Mattsson et al. 1999), whereas aux1 mutants exhibit an agravitropic root phenotype (Maher and Martindale 1980).

Auxins are initially synthesized in the shoot apex, then transported to their target tissues. In the root, auxins are transported in both an acropetal (base-to-apex) and basipetal (apex-to-base) direction within inner and outer tissues of the root apex, respectively. Rubery and Sheldrake (1974) and Raven (1975) proposed in their chemiosmotic hypothesis that the polarity of auxin movement is provided by the asymmetric localization of auxin efflux carriers (Rubery and Sheldrake 1974). The asymmetric localization of several AtPIN proteins has been reported recently, consistent with the chemiosmotic hypothesis (Galweiler et al. 1998; Müller et al. 1998). However, the role of the auxin influx carrier during polar auxin transport remains unclear (Parry et al. 2001), prompting us to investigate AUX1 localization in planta. Our studies reveal that AUX1 performs a dual auxin transport function in the Arabidopsis root apex, facilitating acropetal and basipetal auxin transport in protophloem and lateral root cap cells, respectively. We report that to perform this dual transport function, AUX1 exhibits a complex pattern of subcellular localization.

Results and Discussion

\section{Functional epitope-tagged AUX1 proteins facilitate localization studies in the root apex}

Mutant and inhibitor studies have led to the conclusion that the auxin influx carrier regulates root gravitropism (Marchant et al. 1999; Parry et al. 2001). To investigate the signaling function(s) of the auxin influx carrier at the cellular level, AUX1 was localized in the Arabidopsis root apex. The AUX1 protein was visualized by use of an epitope-tagging approach. A single copy of the nine amino acid hemagglutinin (HA) motif was fused to either terminus of AUX1, creating $\mathrm{N}$ and C HA-AUX1 epitopetagged proteins under the control of native $A U X 1$ regulatory sequences, then transformed into the aux1-22 null allele background. Cosegregation studies revealed that one copy of the $\mathrm{N}$ and $\mathrm{C} H A-A U X 1$ transgenes were sufficient to rescue the agravitropic phenotype of the aux1-22 mutant allele (data not shown). Complementa- 
tion of aux1-22 by either transgene confirmed that both versions of the epitope-tagged protein fully replaced the function of endogenous AUX1 in gravitropic signaling. HA-AUX1 localization in transgenic root apical tissues was visualized by use of indirect immunofluorescense microscopy. Both N (Fig. 1) and C (data not shown) HAtagged AUX1 versions were detected in identical patterns within a discrete subset of stele, columella, lateral root cap, and epidermal cells.

\section{AUX1 protophloem localization defines a novel auxin transport pathway in roots}

HA-AUX1 was detected within two discrete cell files of the stele (Fig. 1B) as well as in stele initials (Fig. 1H). A radial section through a GUS-stained $A U X 1$ :: uidA root apex (Fig. 1E) illustrates stelar AUX1 expression in both protophloem poles (Fig. 1D). The transition of protophloem cells to mature phloem in roots involves the formation of a sieve plate and occurs in the region of maximum growth rate (Esau 1965). The epitope-tagged protein and the GUS reporter are detected in the basal half of the elongation zone (Fig. 1B,C), indicating that AUX1 stele expression is limited to protophloem cells.

To date, polar auxin transport in the xylem parenchyma has been assumed to represent the sole conduit for IAA transport to roots (Palme and Gälweiler 1999). However, significant concentrations of free IAA $(\sim 1 \mu \mathrm{M})$ have been detected in the phloem, although its physiological significance is unclear (Baker 2000). Nevertheless, the cellular pathway for post-phloem transport in roots remains to be established (Bret-Harte and Silk 1994; Fisher and Oparka 1996). Protophloem cell files represent a likely route, but these cells would require carriers to unload IAA from the phloem, as they retain an intact PM prior to sieve plate formation (Esau 1965). In this respect, localization of HA-AUX1 in the protophloem cell PM is significant. Interestingly, HA-AUX1 localization in the protophloem cell PM appeared asymmetric (Fig. 1F). Localization of AUX1 to the upper PM of protophloem cell files (Fig. 1F) would facilitate the acropetal, post-phloem
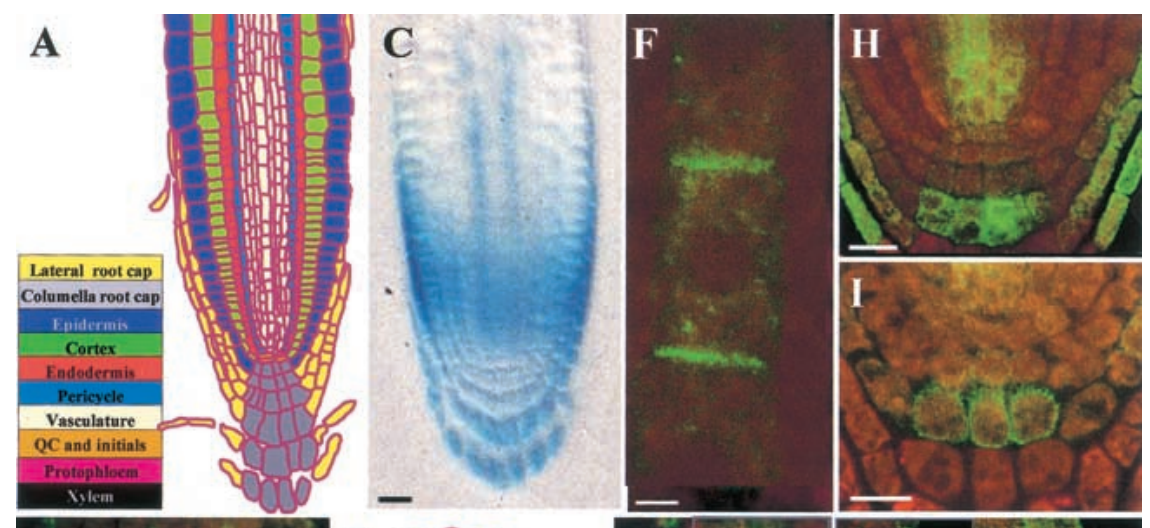
movement of IAA, prompting us to investigate its subcellular distribution further. Separation of the protophloem PM from its cell wall prior to detection revealed that PM HAAUX1 was associated with the upper membrane (Fig. 1G). Double-labeling experiments designed to localize HAAUX1 relative to the asymmetric root stele marker, AtPIN1 (J. Friml and K. Palme, in prep.) revealed that the proteins were preferentially targeted to the upper and lower protophloem PM domains, respectively (Fig. $1 \mathrm{G}$ and in-
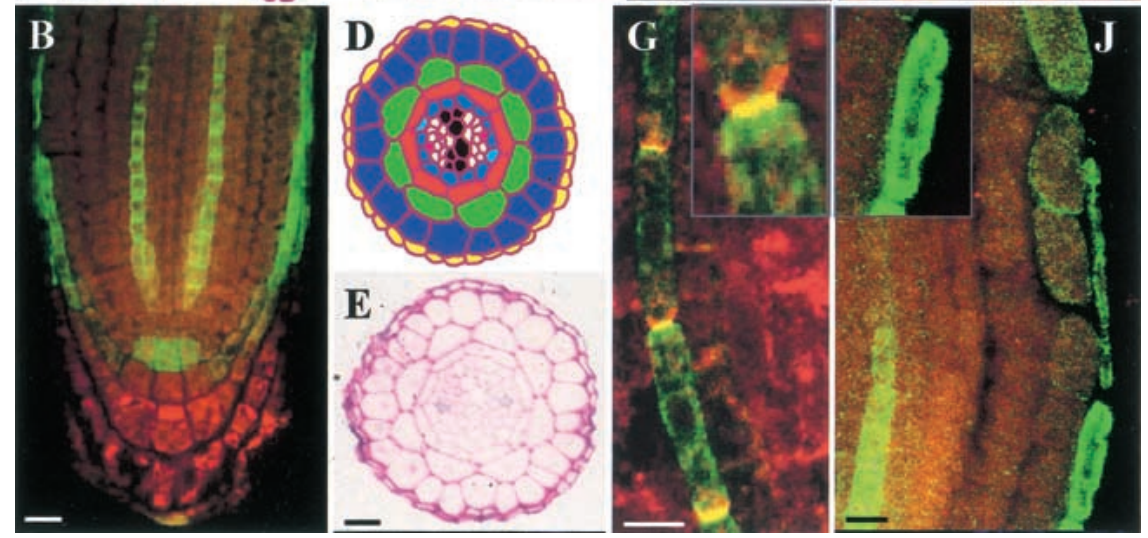

Figure 1. AUX1 is expressed in a subset of columella, lateral root cap, and stele tissues. (A) Schematic of Arabidopsis root apical tissue organization. (Yellow) Lateral root cap; (gray) columella; (royal blue) epidermis; (green) cortex; (red) endodermis; (turquoise) pericycle; (white) vasculature; (orange) QC and initials (Marchant et al. 1999). (B) Immunolocalization of HA-AUX1 in the root apex using confocal microscopy. (C) DIC image of whole-mount GUS-stained AUX1:: uidA root apex. (D) Schematic of root radial tissue organization highlighting protophloem (magenta) and protoxylem (black) poles. $(E)$ Radial section of GUS-stained AUX1:: uidA root illustrating protophloem expression. $(F)$ Asymmetric immunolocalization of HA-AUX1 in protophloem cell file. $(G)$ Dual labeling of HA-AUX1 (green) and AtPIN1 (red) in protophloem cell file. $(H)$ Immunolocalization of HA-AUX1 in stele initials/S2 layer. (I) Immunolocaliation of HA-AUX1 in S2 PM. ( $J$ ) Immunolocalization of HA-AUX1 in LRC (main and inset) and CEZ (main) epidermal cells. Scale bars for $B, C, E, H, I, 20 \mu \mathrm{m}_{i ;} F, 2 \mu \mathrm{m}$, and $G, J, 10 \mu \mathrm{m}$. set).

\section{The aux1 mutation disrupts IAA} accumulation at the root apex

The asymmetric localization of AUX1 and AtPIN1 to the upper and lower plasma membranes of protophloem cell files (Fig. 1G) would be expected to facilitate the acropetal, post-phloem movement of IAA. On the basis of this model, mutations in AUX1 would be predicted to disrupt post-phloem IAA unloading in the root apex. To test this possibility, IAA levels were measured directly by use of high-resolution gas chromatography-selected reaction-monitoring mass spectrometry (GC-SRM-MS) in the apical $5 \mathrm{~mm}$ of wild-type and aux1 Arabidopsis roots divided into 1-mm segments. Wild-type root tissues accumulated highest IAA levels in the most apical $1-\mathrm{mm}$ segment (Fig. 2A; students $t$ test, $P=0.022$ ). In contrast, aux1 root apical tissues failed to accumulate IAA at a significantly higher level than in more basal segments (Fig. 2A; $P=0.19$ ). Direct 

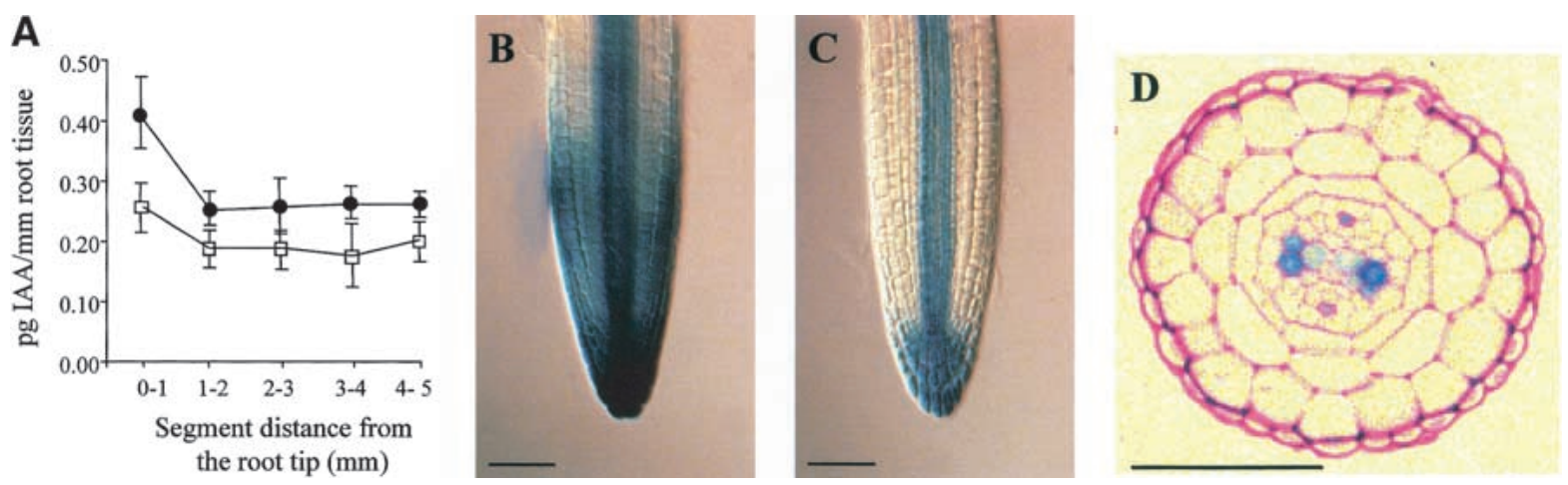

Figure 2. Analysis of IAA accumulation in wild-type and aux1 Arabidopsis root apices. (A) High resolution IAA quantitation in Arabidopsis wild-type $(-)$ and aux1 ( $\square$ ) root segments. (B) DIC image of whole-mount GUS-stained wild-type IAA2::uidA root apex. (C) DIC image of whole-mount GUS-stained aux1 IAA2:: uidA root apex. (D) Radial section of GUS-stained wild-type IAA2:: uidA root apex. Scale bars, $50 \mu \mathrm{m}$.

comparison of IAA levels in the apical $1 \mathrm{~mm}$ of wild-type and aux 1 roots highlighted the reduced capacity of mutant root apical tissues to accumulate IAA (Fig. 2A; $P=0.012$ ) consistent with a phloem-unloading defect. On the basis of these observations, we propose that AUX1 unloads IAA from the mature phloem, then delivers the hormone directly to the root meristem via the protophloem cell files (Fig. 1B).

\section{AUX1 localization in gravity-sensing columella cells appears dynamically regulated}

Our localization studies revealed that AUX1 is expressed in selected columella cells (Fig. 1B). The columella tissue represents the site of gravity perception in the root apex (Blancaflor et al. 1998). Gravity perception is mediated by starch granules (statoliths) that sediment in response to a gravity stimulus. Localization of HA-AUX1 to the columella tissue suggests that the auxin permease facilitates IAA redistribution following a gravitropic stimulus.

AUX1 expression is limited to a single tier of statolithcontaining columella cells (Fig. 1B,H,I). The three tiers of statolith-containing cells (termed S1-S3) arise after repeated divisions of the columella initials. Subsequent divisions of columella initials introduce new tiers of cells that displace the proceeding tier toward the root tip (Fig. 1A). HA-AUX1 was limited to the S2 tier in the majority of roots analyzed (Fig. 1H). However, in a minority of roots, HA-AUX1 was located in the S1 layer (Fig. 1B), reflecting that AUX1 is first detected as columella cells undergo the S1 to S2 transition.

Laser ablation experiments have shown the importance of the S1 and S2 layers for gravity perception (Blancaflor et al. 1998). The strict temporal expression of HAAUX1 in the S1/S2 layers may have important functional significance for gravitropism. Acting as an auxin permease, AUX1 would enable the central tier of gravitysensing columella cells to accumulate sufficient IAA to initiate a gravitropic response. Closer inspection of the subcellular distribution of AUX1 in S2 cells revealed an apparently dynamic pattern of localization. In $>80 \%$ of roots analyzed, the HA-AUX1 signal was associated with the S2 cell cytoplasm (Fig. 1H). However, in $\sim 10 \%$ of roots, S2 HA-AUX1 was observed at the cell periphery (Fig. 1I) or was undetectable in the remainder (data not shown). Cells were imaged at different optical depths to confirm localization. Moreover, our observations are consistent with subcellular fractionation experiments on wild-type Arabidopsis roots (R. Swarup, unpubl.).

The apparent variability of HA-AUX1 S2 subcellular localization and detection may indicate dynamic regulation of PM targeting and turnover, respectively. Functionally, if the gravity perception machinery regulates AUX1 PM targeting, a gravitropic stimulus may cause S2 cells to transiently accumulate sufficient IAA to initiate a gravitropic response. Significantly, differences in the intensity of GUS staining have been observed between wild-type and aux1 columella tissues expressing the auxin inducible reporter IAA2::uidA (Fig. 2B,C). Our results suggest that AUX1, and hence auxin, are involved during the early stages of gravity signal transduction.

\section{AUX1 facilitates basipetal auxin transport}

LRC cells are proposed to facilitate the basipetal redistribution of IAA from the columella to epidermal cells (Estelle 1996). LRC cells overlay the gravitropic response tissues of the distal elongation zone (DEZ), the initial site of root gravitropic curvature (Fig. 1A; Ishikawa and Evans 1993). HA-AUX1 is expressed in the lateral root cap (LRC; Fig. 1B,J) and epidermal cells that have lost contact with the LRC and are entering the central elongation zone (CEZ; Fig. 1J). The LRC/CEZ patterns of expression suggest that AUX1 may facilitate basipetal transport of IAA in these tissues. To test whether aux 1 is defective for basipetal auxin transport, the spatial expression pattern of the auxin-responsive IAA2:: uidA reporter gene was compared in a mutant versus wild-type background. Whereas IAA2::uidA was strongly expressed in protoxylem poles of both genetic backgrounds (Fig. 2B-D), clear differences in GUS staining were observed between wild-type and mutant LRC/DEZ/ CEZ tissues (Fig. 2B,C). The absence of detectable IAA2:: uidA expression in aux1 LRC/DEZ/CEZ tissues (Fig. 2C) is consistent with the mutation disrupting basipetal auxin transport (Rashotte et al. 2001). Our results suggest that the aux 1 agravitropic root phenotype results from either disruption of basipetal auxin transport, and/ or the loss of AUX1 expression in gravity-sensing columella cells. We are currently expressing AUX1 in discrete subdomains of the aux1 root apex to clarify this point. 
The aux1 root agravitropic phenotype can be rescued by the lipophilic synthetic auxin, 1-NAA (Yamamoto and Yamamoto 1998; Marchant et al. 1999; Fig. 3C,E). In contrast, auxins that require influx carrier activity such as 2,4D are unable to rescue aux1 root gravitropism (Fig. $3 \mathrm{G}, \mathrm{I}$ ). The ability of a membrane permeable auxin (Delbarre et al. 1996) to bypass the auxin uptake defect of the mutant and fully restore aux1 gravitropism led Marchant et al. (1999) to conclude that AUX1 must play a permissive (rather than regulatory) function during gravitropism. The nonasymmetric localization of AUX1 in lateral root cap cells (Fig. 1J) is consistent with this conclusion, in which the auxin influx carrier would serve only to permeabilize (rather than actively direct) the redistribution of auxin following a gravitropic stimulus. Instead, the polarity of basipetal auxin transport is likely to be regulated by the apical PM localization of the auxin efflux carrier component, AtPIN2, in LRC cells (J. Friml and K. Palme, unpubl.). As a substrate of the auxin efflux carrier (Delbarre et al. 1996), 1-NAA should be able to move basipetally from LRC cell to cell. We directly tested whether 1-NAA mediated rescue of aux1 root gravitropism resulted from the restoration of basipetal auxin transport by use of the aux1 IAA2::uidA line. We observed that the restoration of aux1 root gravitropism by $10^{-7} \mathrm{M} 1$-NAA (Fig. $3 \mathrm{E}$ ) coincided with the induction of GUS activity in DEZ/CEZ tissues (Fig. 3F), indicative of basipetal auxin transport activity. In contrast, $10^{-8} \mathrm{M}$ 1-NAA failed to elicit either response (Fig. $3 \mathrm{C}, \mathrm{D})$. We conclude that the dose-dependent relationship between 1-NAA rescue of aux1 gravitropism (Fig. $3 \mathrm{E})$ and the restoration of IAA2::uidA expression in mutant LRC/DEZ/CEZ tissues (Fig. 3F) provides further evidence to support the importance of basipetal auxin transport for root gravitropic signal transduction (Rashotte et al. 2000).

The inability of aux1 LRC cells to deliver auxin to the rapidly growing cells of the DEZ/CEZ represents a likely basis for the mutant agravitropic root phenotype. This defective auxin redistribution model is also likely to explain the auxin-resistant root growth phenotype of the

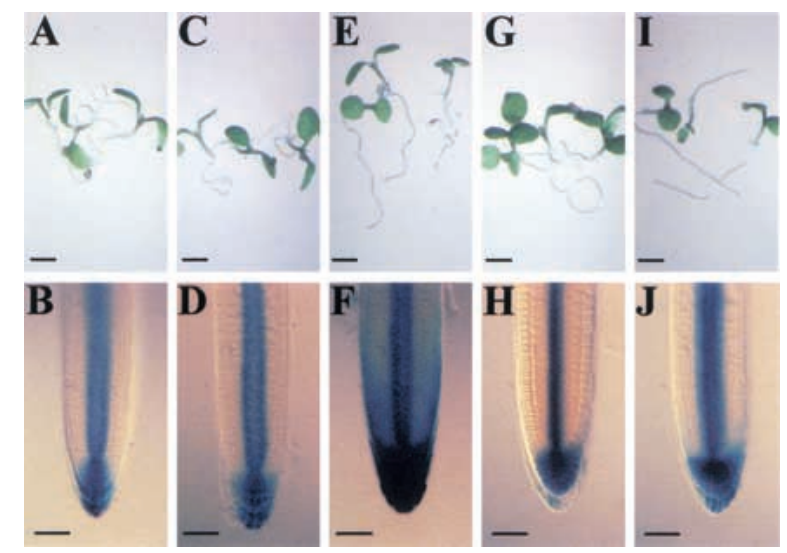

Figure 3. Restoration of aux1 gravitropism by 1-NAA coincides with basipetal auxin redistribution. Root gravitropic phenotype of aux1-100 $(A, C, E, G, I)$ and corresponding IAA2::uidA reporter expression pattern $(B, D, F, H, J)$ when grown in the absence $(A, B)$ or presence of $10^{-8} \mathrm{M} 1$-NAA $(C, D), 10^{-7} \mathrm{M} 1$-NAA $(E, F), 10^{-8} \mathrm{M} 2,4 \mathrm{D}(G, H)$, or $10^{-7} \mathrm{M} 2,4 \mathrm{D}(I, J)$. Scale bars in $A, C, E, G, I, 2 \mathrm{~mm} ; B, D, F, H, J, 50 \mu \mathrm{m}$. aux1 mutant (Maher and Martindale 1980). The mutant exhibits a reduced level of sensitivity toward the auxins $2,4 \mathrm{D}$ and IAA that represent substrates of the auxin influx carrier (Delbarre et al. 1996), whereas retaining a wild-type level of sensitivity toward the lipophilic auxin, 1-NAA (Yamamoto and Yamamoto 1998; Marchant et al. 1999). To determine the basis for the differential auxin sensitivity of the mutant, aux1 IAA2: uidA seedlings were grown in the presence of the $10^{-8}$ and $10^{-7} \mathrm{M}$ 1-NAA and $2,4 \mathrm{D}$. At a $10^{-8} \mathrm{M}$ concentration, neither auxin resulted in a significant retardation of aux1 root growth or induced IAA2:: uidA reporter expression (Fig. $3 \mathrm{D}, \mathrm{H})$ relative to the untreated control (Fig. $3 \mathrm{~B}$ ). In contrast, $10^{-7} \mathrm{M} 1$-NAA caused $\sim 30 \%$ root growth inhibition (data not shown) and was associated with the induction of IAA2: : uidA expression in LRC/DEZ/CEZ tissues (Fig. 3F). However, an equivalent concentration of 2,4D caused no root growth retardation (data not shown) and failed to induce further IAA2::uidA expression (Fig. 3J). Our results imply that the aux12,4D resistant root growth phenotype results from the inability of mutant LRC cells to deliver the auxin to, and therefore repress, the elongation of DEZ/CEZ cells.

\section{Implications}

The results presented here provide new insight into several key aspects of auxin transport. Our results question the traditional view that polar auxin transport represents the long-distance IAA transport pathway in plants. We conclude from MS measurements (Fig. 2A) that a significant proportion of root apical IAA is unloaded by AUX1 from a phloem source. The localization of AUX1 in the protophloem poles (Fig. 1B,C,E) has revealed a novel apoplastic transport pathway for delivering phloem-borne IAA to the root apex. The asymmetric distribution of influx (and efflux) carrier proteins (Fig. 1G) in protophloem pole cells would promote the acropetal movement of IAA to the root apex. Our results are likely to provide a model for other phloem-mobilized constituents such as sucrose, whose mechanism of unloading at the root apex remains controversial (Bret-Harte and Silk 1994; Fisher and Oparka 1996). In addition to its novel phloem-related unloading function, we have shown that AUX1 facilitates auxin transport in columella/LRC/CEZ tissues (Fig. 2). Therefore, AUX1 performs a dual function, facilitating acropetal and basipetal auxin transport within inner and outer tissues of the root apex, respectively.

Our study has revealed a complex pattern of AUX1 subcellular localization (Fig. 1). Protophloem pole cells target AUX1 to their upper PM surface (Fig. 1F,G), whereas no evidence of polar localization is apparent in LRC cells (Fig. 1J, see inset). Similarly, the constitutive polar trafficking of AUX1 in protophloem pole cells contrasts the apparent dynamic pattern of AUX1 localization in columella cells (Fig. 1H,I). This complex pattern of AUX1 targeting in different root cell types contrasts the basal localization of AtPIN1 in Arabidopsis inflorescence and post-midglobular stage embryo cells /Gälweiler et al. 1998; Steinmann et al. 1999). Our colocalization results have revealed that AUX1 and AtPIN1 are targeted to apical and basal ends of protophloem cells (Fig. 1G), suggesting that the targeting of each auxin carrier is regulated distinctly, in agreement with earlier physiological studies (Delbarre et al. 1998; Morris and Robinson 1998). However, antibody-based studies have 
observed recently that the vesicular trafficking inhibitor Brefeldin A is capable of disrupting AUX1 and AtPIN1 targeting (Steinmann et al. 1999; J. Friml and M. Bennett, unpubl.). Both proteins therefore appear to represent substrates of the polar membrane trafficking system. Genetic screens designed to identify mutations that disrupt targeting of auxin carriers are in progress (Steinmann et al. 1999; Sieberer et al. 2000; R. Swarup and M.J. Bennett, unpubl.). Given the diverse pattern of localization described in this work (Fig. 1), AUX1 provides an ideal marker to study the underlying mechanisms that regulate protein targeting, and hence cell polarity, in plants.

\section{Materials and methods}

Transformation of epitope-tagged AUX1 transgenes

The 9-amino-acid HA epitope tag was introduced 2 amino acids from either $\mathrm{N}$ or $\mathrm{C}$ terminus in the AUX1 genomic clone pBS4.6BX by use of a PCR-based approach. N and C HA-AUX1 were subcloned into a BIN19based kanamycin-resistant plant transformation vector (Bevan 1984). Transformation of Arabidopsis (Col. aux1-22) was performed by the floral dip method (Clough and Bent 1998). Complementation experiments were performed on segregrating populations (T2 generation) of aux1-22 transformed with $\mathrm{N}$ and C HA-AUX1 transgenes. Bulk segregant analysis on four lines segregating 3:1 for root gravitropism showed that the $\mathrm{N}$ and C HA-AUX1 transgenes were present in only gravitropic seedlings.

\section{Immunolocalization of AUX1 and AtPIN1 proteins}

The AUX1 immunolocalization and visualization was performed as described in Müller et al. (1998). Fixed and permeabilized 4-day-old Arabidopsis seedlings were incubated with an anti-HA antibody (Boehringer, 1:200) and subsequently with an anti-rat Oregon green-coupled secondary antibody (Molecular Probes, 1:200). To visualize AUX1 at the PM (Fig. 1F), following immunolocalization, roots were partially dehydrated in $60 \%$ ethanol for $30 \mathrm{~min}$, and cells in protophloem files were mechanically detached. For AtPIN1 and HA-AUX1 colocalization, a specific rabbit polyclonal antibody (Gälweiler et al. 1998) was used (1:100) together with a rat anti-HA antibody (Boehringer, 1:200). The primary antibodies were visualized using a mixture of TRITC-coupled anti-rabbit IgG (Sigma, 1:200) and oregon green anti-rat IgG (Molecular Probes, 1:200).

\section{Expression studies using the GUS reporter}

The AUX1::uidA reporter construct was generated as described in Marchant et al. (1999). The IAA2:: uidA reporter gene was constructed by fusing the uidA-coding sequence to the -424 to +1 IAA2 promoter sequence in a binary vector, which was then transformed into wild-type Ws plants (Clough and Bent 1998). A single-copy line was selected and outcrossed into an aux1 mutant background (aux1-100). AUX1:: vidA and IAA2:: uidA seedlings were stained for GUS activity (Willemsen et al. 1998 ) for 3 h, cleared (Malamy and Benfy 1997), and mounted in 50\% glycerol. Root tissues for sectioning were fixed, dehydrated, and embedded in Technovit 7100 resin (Scheres et al. 1994). Then, 4- $\mu \mathrm{m}$ sections were dried onto glass slides, stained with $0.05 \%$ ruthenium red solution for $8 \mathrm{~min}$, and mounted in DePeX (Merck Ltd.) prior to photography.

\section{MS-based IAA analysis}

Root tips were cut in 1-mm sections and collected in $0.05 \mathrm{M}$ phosphate buffer (pH 7.0). For each sample, 50 root sections were pooled. Samples were extracted, purified, and analyzed by GC-SRM-MS (Edlund et al. 1995). Calculation of isotopic dilution was based on the addition of 100 pg of $\left[{ }^{13} \mathrm{C}_{6}\right] \mathrm{IAA} /$ sample.

\section{Acknowledgments}

We thank Ben Scheres and Marcus Grebe for critically reading the manuscript, Burkhard Schulz for providing advice about the HA epitope tag, and Denis Baker for valuable discussion. This work was funded by the BBSRC and European Commission grants to the LATIN and POPWOOD research consortia.

The publication costs of this article were defrayed in part by payment of page charges. This article must therefore be hereby marked "adver- tisement" in accordance with 18 USC section 1734 solely to indicate this fact.

\section{References}

Baker, D.A. 2000. Long-distance vascular transport of endogenous hormones in plants and their role in source: Sink regulation. Israel $\mathrm{J}$. Plant Sci. 48: 199-203.

Bennett, M.J., Marchant, A., Green, H.G., May, S.T., Ward, S.P., Millner, P.A., Walker, A.R., Schultz, B., and Feldmann, K.A. 1996. Arabidopsis AUX1 gene: A permease-like regulator of root gravitropism. Science 273: 948-950.

Bevan, M. 1984. Binary Agrobacterium vectors for plant transformation. Nucleic Acids Res. 12: 8711-8721.

Blancaflor, E.B., Fasano, J.M., and Gilroy, S. 1998. Mapping the functional roles of cap cells in the response of Arabidopsis primary roots to gravity. Plant Physiol. 116: 213-222.

Bret-Harte, M.S. and Silk, W.K. 1994. Nonvascular, symplasmic diffusion of sucrose cannot satisfy the carbon demands of growth in the primary root tip of Zea mays L. Plant Physiol. 105: 19-33.

Casimiro, I., Marchant, A., Bhalerao, R.P., Beeckman, T., Dhooge, S., Swarup, R., Graham, N., Inze, D., Sandberg, G., Casero, P.J., et al. 2001. Auxin transport promotes Arabidopsis lateral root initiation. Plant Cell 13: 843-852.

Chen, R., Hilson, P., Sedbrook, J., Rosen, E., Caspar, T., and Masson, P. 1998. The Arabidopsis thaliana AGRAVITROPIC 1 gene encodes a component of the polar-auxin-transport efflux carrier. Proc. Nat1. Acad. Sci. 95: 15112-15117.

Clough, S.J. and Bent, A.F. 1998. Floral dip: A simplified method for Agrobacterium-mediated transformation of Arabidopsis thaliana. Plant J. 16: 735-743.

Davies, P.J. 1995. Plant hormones: Physiology, biochemistry and molecular biology. ed. 2, Kluwer, London, UK.

Delbarre, A., Müller, P., Imhoff, V., and Guern, J. 1996. Comparison of mechanisms controlling uptake and accumulation of 2,4-dichlorophenoxy acetic acid, naphthalene-1-acetic acid, and indole-3-acetic acid in suspension-cultured tobacco cells. Planta 198: 532-541.

Delbarre, A., Müller, P., and Guern, J. 1998. Short-lived and phosphorylated proteins contribute to carrier-mediated efflux, but not to influx, of auxin in suspension-cultured tobacco cells. Plant Physiol. 116: 833-844.

Edlund, A., Eklof, S., Sundberg, B., Moritz, T., and Sandberg, G. 1995. A microscale technique for gas-chromatography mass-spectrometry measurements of picogram amounts of Indole-3-Acetic-Acid in planttissues. Plant Physiol. 108: 1043-1047.

Esau, K. 1965. Vascular differentiation in plants. Holt, Rinehart and Winston, New York, NY.

Estelle. M. 1996. Plant tropisms: The ins and outs of auxin. Curr. Biol. 6: 1589-1591.

Fisher, D.B. and Oparka K.J. 1996. Post-phloem transport: Principles and problems. J. Ex. Bot. 47: 1141-1154.

Gälweiler, L., Guan., C., Müller, A., Wisman, E., Mendgen K., Yephremov, A., and Palme, K. 1998. Regulation of polar auxin transport by AtPIN1 in Arabidopsis vascular tissue. Science 282: 2226-2230.

Ishikawa, H. and Evans, M.L. 1993. The role of the distal elongation zone in the response of maize roots to auxin and gravity. Plant Physiol. 102: 1203-1210.

Liu, C.M., Xu, Z.H., and Chua, N.H. 1993. Auxin polar transport is essential for the establishment of bilateral symmetry during early plant embryogenesis. Plant Cell 5: 621-630.

Lomax, T.L., Muday, G.K., and Rubery, P.H. 1995. Auxin Transport. In Plant hormones: Physiology, biochemistry and molecular biology. ed. 2 (ed. P.J. Davies), pp. 509-530. Kluwer, London, UK.

Luschnig, C., Gaxiola, R.A., Grisafi, P., and Fink, G.R. 1998. EIR1, a root-specific protein involved in auxin transport, is required for gravitropism in Arabidopsis thaliana. Genes \& Dev. 12: 2175-2187.

Maher, E.P. and Martindale, S.J.B. 1980. Mutants of Arabidopsis-thaliana with altered responses to auxins and gravity. Biochem. Genet. 18: 1041-1053.

Malamy, J.E. and Benfy, P.N. 1997. Organization and cell differentiation in lateral roots of Arabidopsis thaliana. Development 124: 33-44.

Marchant, A., Kargul, J., May, S.T., Müller, P., Delbarre, A., Perrot-Rechenmann, C., and Bennett, M.J. 1999. AUX1 regulates root gravit- 
ropism in Arabidopsis by facilitating auxin uptake within root apical tissues. EMBO J. 18: 2066-2073.

Mattsson, J., Sung, Z.R., and Berleth, T. 1999. Responses of plant vascular systems to auxin transport inhibition. Development 126: 2979-2991.

Morris, D.A. and Robinson, J.S. 1998. Targeting of auxin carriers to the plasma membrane: Differential effects of brefeldin A on the traffic of auxin uptake and efflux carriers. Planta 205: 606-612.

Müller, A., Guan, C., Gälweiler, L., Tänzler, P., Huijser, P., Marchant, A., Parry, G., Bennett, M.J., Wisman, E., and Palme, K. 1998. AtPIN2 defines a locus of Arabidopsis for root gravitropism control. EMBO $J$. 17: 6903-6911.

Okada, K., Ueda, J., Komaki, M.K., Bell, C.J., and Shimura, Y. 1991. Requirement of the auxin polar transport-system in early stages of Arabidopsis floral bud formation. Plant Cell 3: 677-684.

Palme, K. and Gälweiler, L. 1999. PIN-pointing the molecular basis of auxin transport. Curr. Opin. Plant Biol. 2: 375-381.

Parry, G., Delbarre, A., Marchant, A., Swarup, R., Napier, R., PerrotRechenmann, C., and Bennett, M.J. 2001. Novel auxin transport inhibitors phenocopy the auxin influx carrier mutation aux1. Plant $J$. 25: 399-406.

Rashotte, A., Brady, S., Reed, R., Ante, S., and Muday, G. 2000. Basipetal auxin transport is required for gravitropism in roots of Arabidopsis. Plant Physiol. 122: 481-490.

Rashotte, A.M., DeLong, A. and Muday, G.K. 2001. Genetic and chemical reductions in protein phosphatase activity alter auxin transport, gravity response, and lateral root growth. Plant Cell 13: 1683-1697.

Raven, J.A. 1975. Transport of indoleacetic acid in plant cells in relation to $\mathrm{pH}$ and electrical potential gradients and its significance for polar transport. New Phytol. 74: 163-172.

Rubery, P.H. and Sheldrake, A.R. 1974. Carrier mediated auxin transport. Planta 188: 101-121.

Scheres, B., Wolkenfelt, H., Willemsen, V., Terlouw, M., Lawson, E., Dean, C., and Weisbeek, P. 1994. Embryonic origin of the Arabidopsis primary root and root meristem initials. Development 120: 2475 2487.

Sieberer, T., Seifert, G.J., Hauser, M.T., Grisafi, P., Fink, G.R., and Luschnig, C. 2000. Post-transcriptional control of the Arabidopsis auxin efflux carrier EIR1 requires AXR1. Curr. Biol. 10: 1595-1598.

Steinmann, T., Geldner, N., Grebe, M., Mangold, S., Jackson, C.L., Paris, S., Gälweiler, L., Palme, K., and Jurgens, G. 1999. Coordinated polar localization of auxin efflux carrier PIN1 by GNOM ARF GEF. Science 286: $316-318$.

Utsuno, K., Shikanai, T., Yamada, Y., and Hashimoto, T. 1998. AGR, an Agravitropic locus of Arabidopsis thaliana, encodes a novel membrane-protein family member. Plant Cell Physiol. 39: 1111-1117.

Willemsen, V., Wolkenfelt, H., Vrieze, G., Weisbeek, P., and Sheres, B. 1998. The HOBBIT gene is required for formation of the root meristem in the Arabidopsis embryo. Development 125: 521-531.

Yamamoto, M. and Yamamoto, K. 1998. Differential effects of 1-naphathaleneacetic acid and 2,4-dichlorophenoxyacetic acid on the gravitropic response of roots in an auxin resistant mutant of Arabidopsis, aux1. Plant Cell Physiol. 39: 660. 


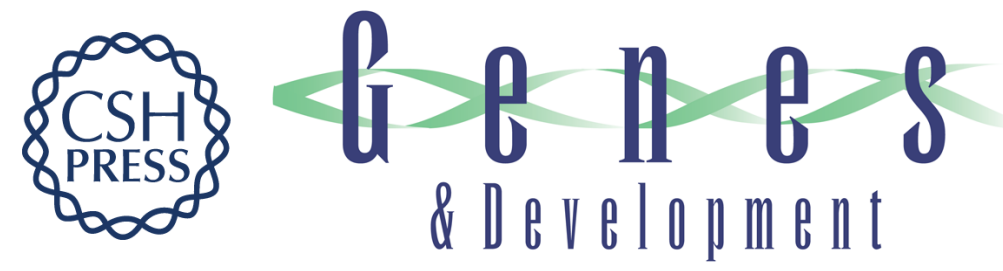

\section{Localization of the auxin permease AUX1 suggests two functionally distinct hormone transport pathways operate in the Arabidopsis root apex}

Ranjan Swarup, Jiri? Friml, Alan Marchant, et al.

Genes Dev. 2001, 15:

Access the most recent version at doi:10.1101/gad.210501

\begin{tabular}{cl} 
References & $\begin{array}{l}\text { This article cites } 35 \text { articles, } 21 \text { of which can be accessed free at: } \\
\text { http://genesdev.cshlp.org/content/15/20/2648.full.html\#ref-list-1 }\end{array}$ \\
License & $\begin{array}{c}\text { Receive free email alerts when new articles cite this article - sign up in the box at the top } \\
\text { Service } \\
\text { right corner of the article or click here. }\end{array}$ \\
\hline
\end{tabular}

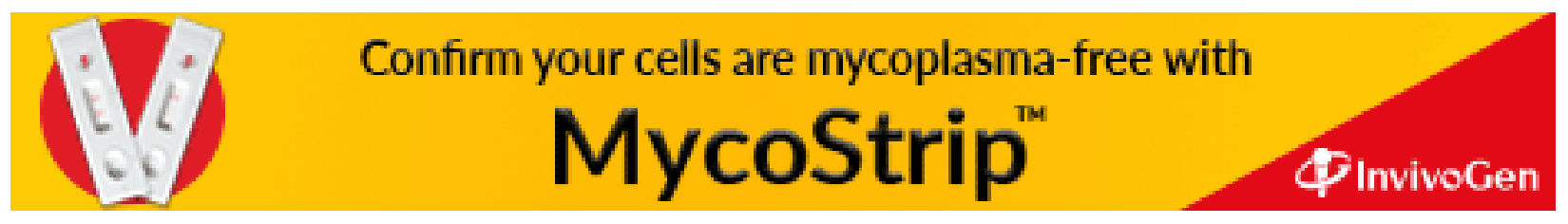

